\title{
Gastric Cancer Presenting as Isolated Ascites: A Diagnostic Challenge
}

\author{
Inês Duarte, Cristina Outerelo \\ Division of Nephrology and Renal Transplantation, Department of Medicine,Centro Hospitalar Universitário Lisboa Norte, Lisbon, Portugal
}

Received: 01/05/2019

Accepted: 08/05/2019

Published: $21 / 06 / 2019$

How to cite this article: Duarte I, Outerelo C. Gastric cancer presenting as isolated ascites: a diagnostic challenge. EJCRIM 2019;6:

doi:10.12890/2019_001141.

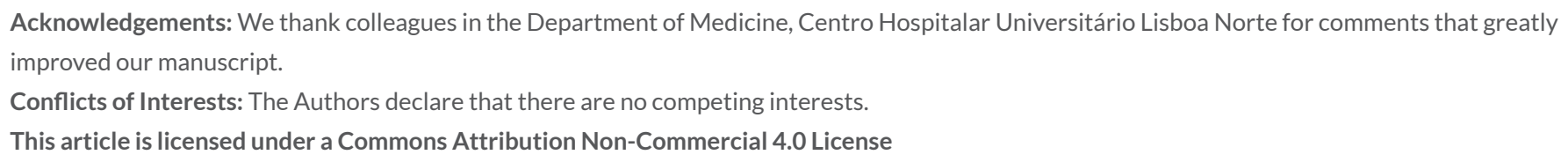

\section{ABSTRACT}

Malignant ascites is a rare first manifestation of gastric carcinoma and is usually associated with symptoms which include early satiety, abdominal pain and deteriorating clinical state. The authors describe the case of a male patient presenting with malignant ascites of rapid onset which was the sole presentation of gastric cancer, highlighting the importance of upper gastric endoscopy even in the absence of gastrointestinal symptoms.

\section{LEARNING POINTS}

- Malignant ascites is present in $10 \%$ of cancer patients and is associated with a poor prognosis.

- Gastric cancer causes malignant ascites in $18 \%$ of cases but is rarely the first symptom.

- As the absence of lesions on a full body CT scan does not exclude gastric cancer, gastrointestinal endoscopy is still the best diagnostic test.

\section{KEYWORDS}

Gastric cancer, ascites, upper endoscopy, poor prognosis

\section{INTRODUCTION}

Ascites is the accumulation of fluid in the peritoneal cavity, and is associated with multiple pathologies, most frequently chronic liver disease. Malignant ascites may result directly from the malignant process or may be secondary to an unrelated comorbidity. It is associated with poor prognosis and contributes to dyspnoea, early satiety, fatigue and abdominal pain.

\section{CASE DESCRIPTION}

A 72-year-old Caucasian man was admitted with lower extremity oedema, weight gain and a progressive increase in his abdominal perimeter over 2 weeks. He denied fever, loss of appetite, nausea, vomiting, diarrhoea or obstipation. He had a history of hypertension, diabetes mellitus, dyslipidaemia, ischaemic heart disease, cerebrovascular disease and heavy smoking (50 pack-years), which had ceased 3 years previously.

Physical examination revealed jugular vein distention at $45^{\circ}$ and hepatojugular reflux, abolished breath sounds in both pulmonary bases, ascites and bilateral lower extremity oedema, without signs or symptoms of chronic liver disease. Laboratory results showed discrete normocytic anaemia $(\mathrm{Hb} 11 \mathrm{~g} / \mathrm{dl})$, leucocytosis $\left(11.25 \times 10^{9} / \mathrm{l}\right)$ with neutrophilia $\left(8.03 \times 10^{9} / \mathrm{l}\right)$ and C-reactive protein $5.6 \mathrm{mg} / \mathrm{dl}$; serum creatinine $1.2 \mathrm{mg} / \mathrm{dl}$, urea $72 \mathrm{mg} / \mathrm{dl}$, total protein $5.3 \mathrm{~g} / \mathrm{dl}$, albumin $2.8 \mathrm{~g} / \mathrm{dl}$ and NT pro-BNP $1295 \mathrm{pg} / \mathrm{ml}$, and proteinuria $126 \mathrm{mg} / 24 \mathrm{~h}$; 
negative serologies for HIV, hepatitis B and C; increased NSE and Cyfra 21-1; carcinoembryonic antigen (CEA), alpha-fetoprotein (AFP) and CA 19.9 within the reference values; and normal thyroid function, transaminases, INR and alkaline phosphatase.

Diagnostic paracentesis revealed a turbid yellow liquid with a serum ascites albumin gradient (SAAG) of $1.1 \mathrm{~g} / \mathrm{dl}$ with total proteins $3.9 \mathrm{mg} /$ dl, LDH $216 \mathrm{U} / \mathrm{l}$, pancreatic amylase $15 \mathrm{U} / \mathrm{l}$ and adenosine deaminase (ADA) 7.4 U/I. The liquid was negative for neoplastic cells.

Full body computed tomography (CT) revealed a $9 \mathrm{~mm}$ solid nodule with irregular contours in the upper lobe of the right lung, bilateral pleural effusion, a small homogeneous liver without individualized nodules, and a large amount of ascites, without other alterations. Transthoracic echocardiography (TE) revealed segmental cardiomyopathy with ejection fraction at the lower limit of normal.

Given the small size of the pulmonary nodule, it was unlikely to have caused the ascites so a biopsy was not carried out. The most probable cause of the ascites appeared to be decompensated heart failure. Diuretic therapy was increased but ineffective, so repeated paracentesis was required for symptom relief due to a progressively larger volume of ascites. Upper digestive endoscopy (UDE) revealed extensive gastric neoplasia. A gastric biopsy was performed revealing gastric adenocarcinoma.

\section{DISCUSSION}

In $10 \%$ of cases, ascites can present as a complication of malignancy, most frequently ovarian (37\%), pancreatic (21\%) and gastric (18\%) cancer $^{[1]}$. Diagnostic paracentesis is required to distinguish benign from malignant ascites. Ascitic fluid generally shows a high concentration of proteins, elevated LDH and low SAAG. A positive cytological examination is diagnostic of malignancy, although a negative result does not preclude the diagnosis ${ }^{[2,3]}$. In this case, the typical characteristics were not present even after repeated biochemical and cytological evaluation of the ascitic fluid. Additionally, a SAAG greater than or equal to $1.1 \mathrm{~g} / \mathrm{dl}$ suggests portal hypertension which was considered due to the congestive heart failure. However, failure to improve with targeted therapy indicated another diagnosis.

Radiological studies are also instrumental. CT allows the detection of even small amounts of ascitic fluid and provides information difficult to obtain on ultrasonography ${ }^{[3]}$. A contrast-enhanced CT scan may demonstrate peritoneal lining enhancement or reveal gastric wall thickening in patients with carcinomatosis or inflammatory peritonitis, but these findings were not present in this case. Exploratory laparoscopy may be required when ascites of undetermined origin is present ${ }^{[4]}$. In this case, before performing an exploratory laparoscopy, we opted to carry out a UDE which revealed extensive gastric neoplasia.

Gastric cancer is one of the most common malignant diseases worldwide; the main risk factors include male gender, non-white race and older age. Hosseini et al. reported that the first symptom was gastric pain in $44.4 \%$ of cases, dysphagia in $20.6 \%$, vomiting in $17.5 \%$, anorexia in $11.1 \%$, nausea in $4.8 \%$ and cachexia in $1.6 \%{ }^{[5]}$. A literature review revealed no reports of ascites as the first manifestation of gastric cancer, although it is the first detected sign of intra-abdominal malignancy in $50 \%$ of patients with peritoneal carcinomatosis ${ }^{[6]}$.

Maeda et al. ${ }^{[7]}$ reported malignant ascites as a late manifestation of gastric cancer, often accompanied by a deteriorated clinical state. Despite the prominent ascites in our patient, the absence of other symptoms or clinical deterioration contributed to the diagnostic challenge.

In conclusion, this case highlights the need for a systematic approach to ascites, especially when there are multiple risk factors, which makes diagnosis more challenging. Furthermore, even in the absence of gastrointestinal symptoms or gastric lesions on CT scanning, gastric cancer should be considered as a cause of the ascites.

\section{REFERENCES}

1. Garrison RN, Kaelin LD, Heuser LS. Malignant ascites. Clinical and experimental observations. Ann Surg 1986;203(6):644-651.

2. Sangisetty SL, Miner TJ. Malignant ascites: a review of prognostic factors, pathophysiology and therapeutic measures. World J Gastrointest Surg 2012;4(4):87-95.

3. Sood R. Ascites: diagnosis and management. JIACM 2000;1(1):81-89.

4. Virmani V, Khandelwal A, Sethi M, et al. Neoplastic stomach lesions and their mimickers: spectrum of imaging manifestations. Cancer Imaging 2012;12(1):269-278.

5. Hosseini SN, Mousavinasab SN, Hoghimi H, et al. Delay in diagnosis and treatment of gastric cancer: from the beginning of symptoms to surgery - an Iranian study. Turk $J$ Gastroenterol 2007;18(2):77-81

6. Sangisetty SL, Miner TJ. Malignant ascites: a review of prognostic factors, pathophysiology and therapeutic measures. World J Gastrointest Surg 2012;4(4):87-95.

7. Maeda H, Kobayashi M, Sakamoto J. Evaluation and treatment of malignant ascites secondary to gastric cancer. World J Gastroenterol 2015;21(39):10936-10947. 